\title{
Prevalence and Covariates of Elevated Depressive Symptoms in Rural Memory Clinic Patients with Mild Cognitive Impairment or Dementia
}

\author{
Julie G. Kosteniuk ${ }^{\mathrm{a}}$ Debra G. Morgan ${ }^{\mathrm{a}}$ Megan E. O'Connell ${ }^{\mathrm{b}}$ \\ Margaret Crossley ${ }^{b}$ Andrew Kirk $^{c}$ Norma J. Stewart ${ }^{d}$ \\ Chandima P. Karunanayake ${ }^{a}$ \\ ${ }^{a}$ Canadian Centre for Health and Safety in Agriculture, ${ }^{b}$ Department of Psychology, \\ 'Division of Neurology, College of Medicine, and d College of Nursing, University of Saskatchewan, \\ Saskatoon, Sask., Canada
}

Key Words

Alzheimer's disease · Dementia - Mild cognitive impairment · Depression ·

Depressive symptoms $\cdot$ Rural patients

\begin{abstract}
Background/Aims: To estimate the prevalence, severity, and covariates of depressive symptoms in rural memory clinic patients diagnosed with either mild cognitive impairment (MCI) or dementia. Methods: In a cross-sectional study of 216 rural individuals who attended an interdisciplinary memory clinic between March 2004 and July 2012, 51 patients were diagnosed with MCI and 165 with either dementia due to Alzheimer's disease (AD) or non-AD dementia. The Center for Epidemiologic Studies of Depression Scale (CES-D) was used to estimate the severity and prevalence of clinically elevated depressive symptomatology. Results: The prevalence of elevated depressive symptoms was $51.0 \%$ in the MCI patients and $30.9 \%$ in the dementia patients. Depressive symptoms were more severe in the MCI patients than in the dementia patients. Elevated depressive symptoms were statistically associated with younger age for the MCI group, with lower self-rated memory for the dementia group, and with increased alcohol use and lower quality of life ratings for all patients. In the logistic regression models, elevated depressive symptoms remained negatively associated with selfrated memory and quality of life for the patients with dementia, but significant bivariate associations did not persist in the MCI group. Conclusions: The high prevalence and severity of depressive symptoms among rural memory clinic patients diagnosed with either MCI or dementia warrant continued investigation.




\section{Introduction}

The prevalence of depression tends to be higher and demonstrates greater variability among individuals with diagnosed dementia than among the general older adult population. Specifically, the rates of depressive symptoms among community-dwelling older adults range from 16 to 38\% [1-3] and the rates of subthreshold or 'non-major depression' (minor subsyndromal and recurrent brief depression) range from 9 to $31 \%$ among community-based adults over 55 years of age [4]. In comparison, the prevalence rates of depression range from 3 to $63 \%$ among community-dwelling individuals with mild cognitive impairment (MCI) $[5,6]$ and from 20 to $50 \%$ among individuals with dementia [5, 7].

$\mathrm{MCI}$ is recognized as objectively measured cognitive impairment without accompanying significant functional impairment [8]. An estimated 15\% of individuals aged 65 years and older with MCI progress to dementia annually [9]. Panza et al. [6] reviewed five hypotheses regarding the association between depression and the development of $\mathrm{MCI}$, and the role of depression in the progression of MCI to dementia: (1) MCI and depression may share risk factors for development (e.g. genetic, environmental, vascular); (2) the development of depression may be a reaction to cognitive decline; (3) late-life depressive symptoms may be early symptoms of MCI; (4) depression may be a cause of hippocampal atrophy leading to $\mathrm{MCI}$, and (5) depression may progress to dementia through a genetic link in some individuals. It is probable that the associations between depression, $\mathrm{MCI}$, and dementia are not attributable to a single factor but rather to multiple factors [5, 10-12].

According to recent reviews, the data are inconclusive regarding depression as a risk factor for dementia versus depression as a prodromal symptom of dementia [7, 13]. However, these hypotheses need not be mutually exclusive $[6,11]$. Kasahara et al. [14] and Enache et al. [5] concluded that depression is both a risk factor for dementia due to Alzheimer's disease (AD) and a prodromal state of $\mathrm{AD}$, given the following evidence: (1) depression in midlife is associated with an increased risk of AD development in later life, (2) depression in later life is a precursor or early presenting symptom of $\mathrm{MCI}$ and $\mathrm{AD}$, and (3) depression is associated with a risk of progression to $\mathrm{MCI}$ and $\mathrm{AD}$. For example, Modrego and Ferrandez [15] estimated a 2.6 times greater risk of progression from MCI (amnestic) to AD in patients with major depression (85\%) compared with patients with MCI alone (32\%) in a community-based sample. Additionally, depression in MCI was associated with earlier progression to AD [15].

Although there is a body of evidence regarding the prevalence of depression in individuals with MCI or dementia as detailed in recent reviews $[5,6,16]$, it has been noted that fewer studies have estimated the prevalence of depressive symptoms in subtypes of MCI [6] and types of non-AD dementia [5]. To the best of our knowledge, few studies have investigated the factors associated with depressive symptoms in a rural community-dwelling sample (vs. institutionalized or hospitalized) of memory clinic patients with diagnosed MCI or dementia.

The purposes of the present study were to estimate the severity, prevalence, and associated factors of elevated depressive symptoms in individuals referred to a memory clinic for rural and remote residents and diagnosed with MCI (amnestic, non-amnestic, and unspecified) or dementia (AD and non-AD).

\section{Methods}

\section{Study Population and Sample}

The Rural and Remote Memory Clinic (RRMC) is located in the city of Saskatoon (population 260,600) [17] and serves rural and remote individuals living independently in commu- 
Kosteniuk et al.: Prevalence and Covariates of Elevated Depressive Symptoms in Rural Memory Clinic Patients with Mild Cognitive Impairment or Dementia

nities that are more than $100 \mathrm{~km}$ from Saskatoon or Regina (the two census metropolitan areas in the province of Saskatchewan). Patients and families are referred to the familycentred interprofessional RRMC by their primary care health provider and receive a preclinical telehealth-facilitated assessment by a neuropsychologist and nurse. Four to 6 weeks later, patients and caregiver(s) attend an in-person clinic day evaluation that involves the full clinical team of a neurologist, physical therapist, dietitian, and neuropsychology team. During clinic day evaluation, patients complete a baseline questionnaire consisting of sociodemographic and clinical measures (e.g. behaviour, functional ability, mood, stress, and quality of life) and a standardized neuropsychological battery. They also receive a physical therapy assessment, CT head scan, up-to-date blood work, and a neurological examination. Caregivers complete a separate questionnaire to assess patient behaviour, functional ability, and neuropsychiatric symptoms, as well as caregiver burden, quality of life, and distress. The clinic day evaluation concludes with a case conference by the full clinical team followed by a consultation between the patient, family, neuropsychologist, and neurologist. Further details regarding the operation and clinical assessment processes of the RRMC are provided by Morgan et al. [18].

Between March 2004 and July 2012, the RRMC enrolled 363 patients (the clinic's 6th data release), 306 of whom attended a clinic day evaluation and received a diagnosis. Diagnoses based on the interprofessional information included the following: no cognitive impairment; amnestic MCI (single or multiple domain); non-amnestic MCI (single or multiple domain); vascular cognitive impairment no dementia (VCI); MCI not specified; dementia due to AD, and non-AD dementia including frontotemporal dementia (FTD), dementia with Lewy bodies (DLB), vascular dementia (VaD), dementia due to multiple aetiologies (DME), Parkinson's disease dementia (PD), Huntington's disease dementia (HD), dementia due to normal pressure hydrocephalus (NPH), and cognitive impairment due to a general medical condition. Of these 306 patients, 65 were diagnosed with no cognitive impairment. Of the remaining 241 patients, 25 patients completed 18 or fewer items on the 20-item Center for Epidemiologic Studies of Depression Scale (CES-D) and were therefore not eligible for the present study. The remaining 216 patients were included in the sample.

Due to the small number of patients within the specific diagnostic subgroups, for the purposes of the present study, the patients were assigned to one of two diagnostic groups: MCI ( $n=51)$ or dementia due to AD or non-AD dementia $(n=165)$. Of the 51 patients in the MCI diagnostic group, 20 were diagnosed with amnestic MCI (single or multiple domain), 21 with non-amnestic MCI (single or multiple domain) or VCI, and 10 with MCI not specified as amnestic or non-amnestic. Of the 165 patients included in the dementia diagnostic group for this analysis, 110 were diagnosed with dementia due to AD and 55 with non-AD dementia $(20$ FTD, $12 \mathrm{DLB}, 10 \mathrm{VaD}, 8 \mathrm{DME}, 2 \mathrm{PD}, 1 \mathrm{HD}, 1 \mathrm{NPH}$, and 1 cognitive impairment due to a general medical condition).

\section{Measures}

The outcome measure (CES-D) and independent variables (sociodemographic and clinical measures) included in the present study were collected from the patients during the initial clinic day evaluation. The patients were provided assistance from clinic staff and/or caregivers to complete the various self-report measures, e.g. by clarifying instructions and ensuring that reverse-scored items were properly interpreted.

\section{Outcome Variable}

Depressive symptoms were assessed with the CES-D $[19,20]$. Although originally intended for use in the general population, the 20-item CES-D is a commonly used screening instrument to detect depressive symptoms among older adults and individuals with AD [6, 
7]. Using major depression experienced during the previous month as assessed with the Diagnostic Interview Schedule as the criterion in a study of community-based individuals 55-85 years of age, a CES-D cutoff score of $\geq 16$ had a weighted sensitivity of $100 \%$ and specificity of $88 \%$ with no differences in true and false positives among those with cognitive impairment [21]. The CES-D assesses the frequency and severity of depressive symptoms experienced by an individual during the previous week across four dimensions (i.e. depressed affect, lack of positive affect, somatic complaints, and interpersonal difficulties). Negatively worded items are scored from 0 (rarely or none of the time) to 3 (most or all of the time) and positively worded items are reverse scored in order that higher scale scores (0-60) indicate a higher level of depressive symptomatology. If 1 item from a patient's 20 -item CES-D was missing, the missing item was scored ' 0 '. If $\geq 2$ of the 20 items were missing, the scale for that patient was discarded [20]. The present study used scores of $\geq 16$ to indicate the presence of elevated depressive symptoms as in previous studies of older adults [3,21]. Smarr and Keefer [22] suggest that this cutoff point may be used to identify those at high risk of major depressive disorder. Cronbach's $\alpha$ of 0.86 demonstrated internal consistency reliability for the 20 -item CES-D in the present study.

Patients who did not complete the CES-D had significantly lower $(\mathrm{p}<0.05)$ Modified Mini-Mental State Examination (3MS) scores (61.5) than patients who completed the CES-D (CES-D score $\geq 16$ : $3 \mathrm{MS}$ score 76.5; CES-D score <16: $3 \mathrm{MS}$ score 74.5), indicating that individuals with greater cognitive impairment were less likely to complete the CES-D than individuals with lower cognitive impairment.

\section{Independent Variables}

Sociodemographic measures included gender, age, education, marital status (married or living common-law) and living arrangements (living with others/not living with others). Clinical measures included the following: an indicator of alcohol use, the CAGE (Cutting Down, Annoyance by Criticism, Guilty Feelings, and Eye-Openers questionnaire); the total number of current chronic conditions; the 3MS; self-rating of memory; quality of life (Quality of LifeAlzheimer's Disease scale, QOL-AD), and instrumental activities of daily living (Instrumental Activities of Daily Living scale, IADL).

The CAGE is a 9-item scale with items scored 'yes' (1) or 'no' (0) and total scores ranging from 0 to 9 [23]; a higher CAGE score indicates a higher level of alcohol use. Total number of current chronic conditions ranged from 0 to 22. The 3MS scores range from 0 to 100 based on 15 questions, with lower scores suggesting greater cognitive impairment [24]. The SelfRating of Memory Scale (SRMS) [25] measures current memory ability compared with memory ability 4 years previously, with higher scores suggesting greater current memory ability. The scale consists of 15 items scored on a 5-point scale from -2 to +2 , with total scores ranging from -30 to +30 . Quality of life was measured with the self-rated QOL-AD instrument, with higher scores suggesting higher current patient quality of life [26]. The QOL-AD instrument contains 13 items rated on a 4-point scale as 1 (poor), 2 (fair), 3 (good), or 4 (excellent), with total scores ranging from 13 to 52 . The IADL measure is a self-rated 9-item scale with each item scored as 1 (total dependence), 2 (some assistance required), or 3 (total independence) [27]. Total IADL scores range from 9 to 27; higher scores indicate higher independent functioning in terms of daily activities.

In the case of 4 scales (CAGE, SRMS, QOL-AD, and IADL), if a patient's scale was missing $<25 \%$ of the items, the case mean was imputed for those missing items; if a patient's scale was missing $\geq 25 \%$ of the items, the scale for that patient was discarded $[28,29]$. The diagnostic subgroups of the MCI group (MCI amnestic, MCI non-amnestic, and MCI not specified) and the dementia group (dementia due to $\mathrm{AD}$ and non-AD dementia) were also included as independent variables. 
Table 1. Sociodemographic and clinical characteristics of RRMC patients by diagnostic group

\begin{tabular}{|c|c|c|c|c|c|c|c|c|}
\hline & $\begin{array}{l}\text { Total } \\
(n=216)\end{array}$ & $\begin{array}{l}\text { Sample } \\
\text { size, n }\end{array}$ & $\begin{array}{l}\text { MCI } \\
(n=51)\end{array}$ & $\begin{array}{l}\text { Sample } \\
\text { size, } n\end{array}$ & $\begin{array}{l}\text { Dementia } \\
(\mathrm{n}=165)\end{array}$ & $\begin{array}{l}\text { Sample } \\
\text { size, } n\end{array}$ & $\begin{array}{l}\mathrm{p} \\
\text { value }^{\mathrm{a}}\end{array}$ & $\begin{array}{l}\text { Cohen's } \\
\text { d }\end{array}$ \\
\hline Female, n (\%) & $129(59.7)$ & 216 & $30(58.8)$ & 51 & $99(60.0)$ & 165 & 0.881 & 0.13 \\
\hline Age, years & $73.4 \pm 9.4$ & 215 & $69.8 \pm 10.5$ & 51 & $74.5 \pm 8.8$ & 164 & 0.007 & 0.50 \\
\hline Education, years & $10.8 \pm 3.0$ & 206 & $11.6 \pm 3.2$ & 48 & $10.5 \pm 2.9$ & 158 & 0.023 & 0.36 \\
\hline Married, n (\%) & $146(67.9)$ & 215 & 37 (72.5) & 51 & $109(66.5)$ & 164 & 0.416 & 0.09 \\
\hline Live alone, n (\%) & $46(21.5)$ & 214 & $10(19.6)$ & 51 & $36(22.1)$ & 163 & 0.707 & 0.12 \\
\hline Chronic conditions, $\mathrm{n}$ & $5.4 \pm 2.7$ & 216 & $5.1 \pm 2.4$ & 51 & $5.4 \pm 2.8$ & 165 & 0.622 & 0.12 \\
\hline CAGE score & $0.41 \pm 1.3$ & 208 & $0.82 \pm 1.8$ & 49 & $0.28 \pm 1.1$ & 159 & 0.012 & 0.36 \\
\hline QOL-AD score & $35.5 \pm 5.7$ & 207 & $35.8 \pm 5.8$ & 48 & $35.5 \pm 5.7$ & 159 & 0.554 & 0.05 \\
\hline IADL score & $22.1 \pm 4.8$ & 208 & $25.0 \pm 2.3$ & 48 & $21.3 \pm 5.1$ & 160 & $<0.001$ & 0.94 \\
\hline SRMS score & $-11.1 \pm 7.5$ & 201 & $-10.7 \pm 7.3$ & 49 & $-11.2 \pm 7.6$ & 152 & 0.988 & 0.07 \\
\hline 3MS score & $75.2 \pm 14.7$ & 208 & $88.0 \pm 7.0$ & 50 & $71.1 \pm 14.2$ & 158 & $<0.001$ & 1.51 \\
\hline
\end{tabular}

Values denote means \pm SD unless specified otherwise. Sample sizes vary due to missing values.

${ }^{a}$ Calculated by the $\chi^{2}$ test for nominal variables and the Mann-Whitney U test for interval variables.

\section{Ethical Considerations}

TheoverarchingRRMCstudy received ethicsapprovalfrom the University ofSaskatchewan Behavioural Research Ethics Board. Patients and caregivers (who also provided proxy consent for the patients) provided their written informed consent before allowing their clinical data to be used for research.

\section{Statistical Analyses}

The statistical analyses employed SPSS version 21.0. The prevalence and severity of depressive symptoms (median, mean) were compared between the MCI and dementia (AD and non-AD) diagnostic groups. Sociodemographic and clinical characteristics of the MCI and dementia groups were compared for significant differences, using the $\chi^{2}$ test for nominal variables and the Mann-Whitney U test for interval variables. Cohen's d effect sizes [30] were also calculated (small: $0.20 \leq \mathrm{d}<0.50$; medium: $0.50 \leq \mathrm{d}<0.80$; large: $\mathrm{d} \geq 0.80$ ). The unadjusted odds ratios (OR) of elevated depressive symptoms (CES-D score $\geq 16$ ) are reported for each independent variable, for the MCI and dementia groups separately. Based on bivariate associations with elevated depressive symptoms, independent variables with a $\mathrm{p}<0.25$ [31] were selected for inclusion in the final multiple logistic regression models. Age was included as a possible confounder in the final models for both groups. The 3 independent variables with the smallest $p$ values, in addition to age, were included in the model for the MCI group so as not to exceed the recommended number of 10 cases per variable [31]. Separate multiple logistic regression analyses for the MCI group (enter method) and dementia group (stepwise forward selection with least likelihood ratio selection) were conducted with elevated depressive symptoms (CES-D score $\geq 16$ ) as the outcome. The Hosmer-Lemeshow $\chi^{2}$ test and the $c$ statistic were used to assess the fit of each final model.

\section{Results}

The characteristics of the patients who completed the CES-D and received a clinical diagnosis are shown in table $1(\mathrm{n}=216)$. The majority of the sample was female $(59.7 \%)$, married $(67.9 \%)$, and lived with others $(79.5 \%)$. The patients were an average of 73.4 years of age 
Table 2. Prevalence of elevated depressive symptoms and severity of depressive symptoms in RRMC patients by diagnostic group

\begin{tabular}{|c|c|c|c|c|}
\hline & $\begin{array}{l}\text { Total } \\
(n=216)\end{array}$ & $\begin{array}{l}\text { MCI } \\
(n=51)\end{array}$ & $\begin{array}{l}\text { Dementia } \\
(\mathrm{n}=165)\end{array}$ & $\begin{array}{l}\mathrm{p} \\
\text { value }\end{array}$ \\
\hline Elevated depressive symptoms ${ }^{\mathrm{a}}, \mathrm{n}(\%)$ & 77 (35.6) & $26(51.0)$ & 51 (30.9) & $0.012^{\mathrm{b}}$ \\
\hline Median CES-D score & 11.0 & 16.0 & 10.0 & \\
\hline Mean CES-D score \pm SD (range) & $13.1 \pm 9.1(0-042)$ & $15.9 \pm 9.0(0-35)$ & $12.2 \pm 9.0(0-42)$ & $0.006^{c}$ \\
\hline
\end{tabular}

${ }^{\mathrm{a}}$ CES-D score $\geq 16 .{ }^{\mathrm{b}} \chi^{2}$ test. ${ }^{\mathrm{c}}$ Mann-Whitney U test.

( $\mathrm{SD}=9.4$; range: $44-89)$ and had an average of 10.8 years of education $(\mathrm{SD}=3.0$; range: 4-20).

Also seen in table 1 , the MCI $(n=51)$ and dementia groups $(n=165)$ were comparable in terms of gender, marital status, living arrangements, the number of chronic conditions, QOL-AD score, and SRMS score. Compared with the MCI group, the dementia group was significantly older $(p=0.007 ; d=0.50)$, had fewer years of education $(p=0.023 ; d=0.36)$, and had a lower level of alcohol use $(p=0.012 ; d=0.36)$. As expected, the dementia group had a lower mean IADL score (lower independent functioning; $p<0.001 ; d=0.94$ ) and a lower mean $3 \mathrm{MS}$ score (greater cognitive impairment; $\mathrm{p}<0.001 ; \mathrm{d}=1.51$ ) than the MCI group.

Table 2 shows that elevated depressive symptoms were significantly more prevalent $(\mathrm{p}=0.012)$ in the MCI group (51.0\%) than in the dementia group (30.9\%). Depressive symptoms were also significantly more severe $(\mathrm{p}=0.006 ; \mathrm{d}=0.41)$ in the MCI group (mean CES-D score: $15.9 \pm 9.0$ ) than in the dementia group (mean CES-D score: $12.2 \pm 9.0$ ).

As shown in the unadjusted OR presented in tables 3 and 4, elevated depressive symptoms were significantly associated $(\mathrm{p}<0.05)$ with younger age in the MCI group (OR $=0.94 ; \mathrm{p}=$ 0.042 ), and with greater alcohol use (MCI: $\mathrm{OR}=2.82, \mathrm{p}=0.011$; dementia: $\mathrm{OR}=1.37, \mathrm{p}=$ 0.012 ) and lower quality of life (MCI: $\mathrm{OR}=0.87, \mathrm{p}=0.037$; dementia: $\mathrm{OR}=0.82, \mathrm{p}<0.001$ ) in both groups. Lower self-rated memory $(\mathrm{OR}=0.88 ; \mathrm{p}<0.001)$ was also significantly associated with elevated depressive symptoms in the dementia group. Elevated depressive symptoms did not differ significantly $(\mathrm{p}<0.05$ ) by MCI subtype (non-amnestic: $57.1 \%$; not specified: 50\%; amnestic: $45 \%$ ) or by type of dementia (non-AD: $38.3 \%$; dementia due to AD: $27.3 \%$ ).

Separate multiple logistic regression analyses were conducted for the MCI and dementia groups. The 3 independent variables with the smallest $\mathrm{p}$ values (gender, CAGE score, and QOL-AD score) were included in the MCI group analysis (table 3). The independent variables associated at the bivariate level $(\mathrm{p}<0.25)$ with elevated depressive symptoms (CAGE score, number of chronic conditions, SRMS score, QOL-AD score, IADL score, and type of dementia) were included in the analysis for the dementia group (table 4). For both groups, age was included as a possible confounder in the final models.

The final models shown in tables 5 and 6 are fully adjusted for all variables included in the model for each diagnostic group. In the adjusted model for the MCI group (table 5), gender, age, CAGE score, and QOL-AD score were not significantly independently associated ( $p>0.05)$ with elevated depressive symptoms. The adjusted model in table 6 shows that significant associations with elevated depressive symptoms among patients in the dementia group were retained for lower self-rating of memory $(\mathrm{OR}=0.92 ; \mathrm{p}=0.012)$ and lower quality of life $(\mathrm{OR}=0.88 ; \mathrm{p}=0.003)$. 
Kosteniuk et al.: Prevalence and Covariates of Elevated Depressive Symptoms in Rural Memory Clinic Patients with Mild Cognitive Impairment or Dementia

Table 3. Prevalence and unadjusted OR of elevated depressive symptoms (CES-D score $\geq 16$ ) in the MCI diagnostic group

\begin{tabular}{|c|c|c|c|c|}
\hline & $\begin{array}{l}\text { Sample } \\
\text { size MCI } \\
(\mathrm{n}=51), \mathrm{n}\end{array}$ & $\begin{array}{l}\text { Elevated depressive } \\
\text { symptoms, } \\
\mathrm{n}(\%) \text { or means } \pm \text { SD }\end{array}$ & $\begin{array}{l}\text { Unadjusted OR } \\
(95 \% \mathrm{CI})\end{array}$ & $\begin{array}{l}\mathrm{p} \\
\text { value }^{\mathrm{a}}\end{array}$ \\
\hline Male & 21 & $13(61.9)$ & $2.13(0.68-6.64)$ & 0.192 \\
\hline Female & 30 & $13(43.3)$ & 1.00 & \\
\hline Age, years & 51 & $66.9 \pm 10.3$ & $0.94(0.89-1.00)$ & 0.042 \\
\hline Education, years & 48 & $11.9 \pm 3.6$ & $1.05(0.89-1.26)$ & 0.560 \\
\hline Married & 37 & $19(51.4)$ & $1.06(0.31-3.61)$ & 0.931 \\
\hline Not married & 14 & $7(50.0)$ & 1.00 & \\
\hline Live with others & 41 & $22(53.7)$ & $1.74(0.43-7.09)$ & 0.439 \\
\hline Live alone & 10 & $4(40.0)$ & 1.00 & \\
\hline CAGE score & 49 & $1.5 \pm 2.3$ & $2.82(0.79-10.09)$ & 0.011 \\
\hline Chronic conditions & 51 & $5.4 \pm 2.5$ & $1.14(0.89-1.46)$ & 0.298 \\
\hline SRMS score & 49 & $-11.4 \pm 8.5$ & $0.98(0.90-1.06)$ & 0.287 \\
\hline QOL-AD score & 48 & $33.8 \pm 6.2$ & $0.87(0.77-0.98)$ & 0.037 \\
\hline 3MS score & 50 & $88.0 \pm 7.6$ & $1.00(0.92-1.08)$ & 0.977 \\
\hline IADL score & 48 & $24.6 \pm 2.4$ & $0.85(0.66-1.11)$ & 0.197 \\
\hline \multicolumn{5}{|l|}{$\mathrm{MCI}$} \\
\hline MCI non-amnestic & 21 & $12(57.1)$ & $1.63(0.47-5.60)$ & 0.737 \\
\hline MCI not specified & 10 & $5(50.0)$ & $1.22(0.27-5.59)$ & \\
\hline MCI amnestic (reference) & 20 & $9(45.0)$ & 1.00 & \\
\hline
\end{tabular}

${ }^{\text {a }}$ Calculated by the $\chi^{2}$ test for nominal variables and the Mann-Whitney $\mathrm{U}$ test for interval variables.

Table 4. Prevalence and unadjusted OR of elevated depressive symptoms (CES-D score $\geq 16$ ) in the dementia diagnostic group (AD and non-AD)

\begin{tabular}{lcccr}
\hline & $\begin{array}{l}\text { Sample size } \\
\text { dementia } \\
(\mathrm{n}=165), \mathrm{n}\end{array}$ & $\begin{array}{l}\text { Elevated depressive } \\
\text { symptoms, } \\
\mathrm{n}(\%) \text { or means } \pm \text { SD }\end{array}$ & $\begin{array}{l}\text { Unadjusted OR } \\
(95 \% \mathrm{CI})\end{array}$ & $\begin{array}{c}\mathrm{p} \\
\text { value }^{\mathrm{a}}\end{array}$ \\
\hline Male & 66 & $20(30.3)$ & $0.95(0.49-1.87)$ & 0.891 \\
Female & 99 & $31(31.3)$ & 1.00 & 0.280 \\
Age, years & 164 & $73.2 \pm 9.5$ & $0.98(0.94-1.01)$ & 0.403 \\
Education, years & 158 & $10.2 \pm 2.7$ & $0.94(0.84-1.07)$ & 0.970 \\
Married & 109 & $34(31.2)$ & $1.01(0.50-2.04)$ & 0.007 \\
Not married & 55 & $17(30.9)$ & $1.24(0.55-2.81)$ & 0.00 \\
Live with others & 127 & $41(32.3)$ & $1.37(0.98-1.92)$ & 0.012 \\
Live alone & 36 & $10(27.8)$ & $1.11(0.99-1.25)$ & 0.074 \\
CAGE score & 159 & $0.5 \pm 1.5$ & $0.88(0.84-0.93)$ & $<0.001$ \\
Chronic conditions & 165 & $6.0 \pm 2.7$ & $0.82(0.76-0.89)$ & $<0.001$ \\
SRMS score & 152 & $-15.6 \pm 8.4$ & $1.00(0.97-1.02)$ & 0.956 \\
QOL-AD score & 159 & $31.8 \pm 6.2$ & $0.94(0.88-1.01)$ & 0.093 \\
3MS score & 158 & $70.5 \pm 14.8$ & $1.65(0.83-3.27)$ & 0.153 \\
IADL score & 160 & $20.2 \pm 5.5$ & 1.00 & \\
Non-AD dementia & 55 & $21(38.2)$ & $30(27.3)$ & \\
Dementia due to AD & 110 & & & \\
\hline
\end{tabular}

${ }^{\text {a }}$ Calculated by the $\chi^{2}$ test for nominal variables and the Mann-Whitney U test for interval variables. 
Table 5. Adjusted OR of elevated depressive symptoms (CES-D score $\geq 16)$ in the MCI diagnostic group $(n=$ 46)

\begin{tabular}{lrlllll}
\hline & B & SE & Wald & OR & $95 \%$ CI & p value \\
\hline Gender (male vs. female) & 0.34 & 0.74 & 0.21 & 1.40 & $0.34-5.98$ & 0.647 \\
Age & -0.05 & 0.04 & 1.36 & 0.96 & $0.88-1.03$ & 0.244 \\
CAGE score & 0.84 & 0.51 & 2.76 & 2.33 & $0.86-6.30$ & 0.097 \\
QOL-AD score & -0.10 & 0.08 & 1.68 & 0.90 & $0.78-1.05$ & 0.196 \\
\hline
\end{tabular}

Five missing cases. Hosmer-Lemeshow $\chi^{2}=5.20$; $\mathrm{p}$ value $=0.635$; c statistic $=0.783$.

Table 6. Adjusted OR of elevated depressive symptoms (CES-D score $\geq 16$ ) in the dementia diagnostic group $(\mathrm{n}=148)$

\begin{tabular}{lcccccc}
\hline & B & SE & Wald & OR & $95 \%$ CI & p value \\
\hline Age & -0.038 & 0.02 & 2.53 & 0.96 & $0.92-1.01$ & 0.122 \\
SRMS score & -0.085 & 0.03 & 6.28 & 0.92 & $0.86-0.98$ & 0.012 \\
QOL-AD score & -0.133 & 0.05 & 8.54 & 0.88 & $0.80-0.96$ & 0.003 \\
\hline
\end{tabular}

Seventeen missing cases. Hosmer-Lemeshow $\chi^{2}=4.18 ; \mathrm{p}$ value $=0.841 ; \mathrm{c}$ statistic $=0.810$.

\section{Discussion}

The prevalence rate of elevated depressive symptoms found in the present study for RRMC patients diagnosed with MCI (51.0\%) falls within the range of prevalence rates of depression (depressive symptoms and types such as minor and major depression) of 3-63\% that have been reported in recent systematic reviews of studies of community-dwelling individuals with MCI $[5,6]$. Our findings are also consistent with those of a previous study that reported depressive symptoms in nearly half of the patients with $\mathrm{MCI}$, as assessed with the Beck Depression Inventory [32].

The prevalence rate of elevated depressive symptoms found among the patients diagnosed with non-AD dementia or dementia due to $\mathrm{AD}(30.9 \%)$ in the present study is consistent with prevalence rates of depression ranging from 20 to $50 \%$ reported in reviews of studies of community-based patients with AD dementia [5, 7]. It is also within the range of 25-66\% reported in four separate studies of community-based populations (vs. institutionalized or hospitalized) with diagnosed dementia that have employed different instruments to assess depressive symptomatology. Specifically, a $25 \%$ prevalence rate of depressive symptoms as assessed with the Cornell Scale for Depression in Dementia (CSDD) was reported in a study of memory clinic patients diagnosed with dementia due to AD [33]; among individuals diagnosed with probable AD dementia, a prevalence rate of $40 \%$ was reported as assessed with the Columbia University Scale for Psychopathology in Alzheimer's Disease (CUSPAD) [34]; a $49 \%$ prevalence rate of depressive symptoms was assessed with the Montgomery-Åsberg Depression Rating Scale (MADRS) among individuals referred to outpatient clinics specializing in geriatrics and older psychiatry diagnosed with a dementia type [35], and a prevalence rate of $66 \%$, also assessed with the MADRS, was reported in a study of memory clinic patients diagnosed with early-onset dementia [36]. 
Kosteniuk et al.: Prevalence and Covariates of Elevated Depressive Symptoms in Rural Memory Clinic Patients with Mild Cognitive Impairment or Dementia

We found that elevated depressive symptoms were significantly $(\mathrm{p}<0.05)$ more prevalent and severe in patients with a diagnosis of MCI (51.0\%) than AD or non-AD dementia (30.9\%). In contrast, van der Mussele et al. [33] found the prevalence of depressive symptoms to be significantly higher among those with AD dementia than those with MCI (25 vs. 16\%). As suggested by the range of depression prevalence rates reported in previous studies, variations in prevalence rates and findings regarding the association between depression and dementia are partly attributable to differences in study design as well as the methods employed to assess depression (e.g. assessment instrument, diagnostic criteria, recall period, and severity of symptoms).

In the present study, the prevalence of elevated depressive symptoms was not significantly different between the MCI subtypes: $57.1 \%$ in non-amnestic MCI, $50.0 \%$ in MCI not specified, and $45 \%$ in amnestic MCI patients. These findings are consistent with the results of a previous study that reported no significant differences in the prevalence of depressive symptoms as assessed with the CSDD among four subtypes of MCI [33]. Johnson et al. [37] reported a greater prevalence of depression as assessed with the Geriatric Depression Scale (GDS-30) among non-amnestic than among amnestic MCI community-based patients (depression: 51.0 vs. 33.0\%), as did Hidaka et al. [38] in a study of amnestic compared with non-amnestic MCI community-based individuals (depression: 35.9 vs. $23.5 \%$ ). However, the statistical significance of these differences was not indicated $[37,38]$.

Elevated depressive symptoms also did not significantly differ between those with non-AD dementia (38.2\%) and those with dementia due to $\mathrm{AD}(27.3 \%)$ in the present study. A recent systematic review found that the depression prevalence in community-based studies varied widely from 16 to $86 \%$ in non-AD dementias [5], and previous studies have reported higher rates of depression among individuals diagnosed with non-AD dementias compared with dementia due to AD. Specifically, higher depression rates were found in patients diagnosed with VaD versus AD [39-42], ischemic vascular disease versus AD [43], and DLB versus $\operatorname{AD}[5,7,35]$. In the present study, the patients with a diagnosis of a non-AD dementia $(n=$ $55)$ were combined with the patients with dementia due to AD $(n=110)$ into one group, because of the small number of patients with non-AD dementia diagnoses. Clearly, the non-AD group was highly heterogeneous, and future research with a larger patient sample for the different types of non-AD dementias is needed.

In the present study, elevated depressive symptoms were significantly associated, at the bivariate level, with younger age in patients diagnosed with MCI. This association did not persist in the final multivariable model for the MCI group. Rosness et al. [36] noted a high prevalence rate of mild and moderate depression (66\%) in their study of early-onset dementia memory clinic patients and posited that this may be related to the hypothesized greater insight that younger memory clinic patients ( $<65$ years) have into their situation. Depressed feelings may be associated with loss of work, benefits, and independence among younger patients, particularly if those patients are supporting families [44].

Significant bivariate associations were also found in the present study between elevated depressive symptoms and increased alcohol use as well as lower quality of life in the MCI and dementia groups. At the bivariate level, lower self-rated memory in the dementia group was also associated with elevated depressive symptoms. For the MCI group, these associations did not persist in the final multivariable model. For the dementia group, significant negative associations with quality of life and self-rated memory persisted in the final multivariable model. These findings underscore the manifestation of key features of depression (poor concentration, change in appetite, and decreasing interest or pleasure) in quality-of-life decline among patients with MCI and dementia.

Factors that were not significantly associated at the bivariate level with elevated depressive symptoms in patients diagnosed with MCI or dementia included sociodemographic (gender, 
education, marital status, and living arrangements) and clinical factors (number of chronic conditions, 3MS score, and IADL score). Consistent with the present study, previous studies reported no relationship between depression and gender among patients with dementia [43] or early-onset dementia [36]. However, the absence of an association between elevated depressive symptoms and education found in the present study is in contrast to a previous study that reported a negative association between depression (major and minor) and education among patients with dementia [43]. The absence of an association between elevated depressive symptoms and the degree of cognitive impairment (3MS score) among patients diagnosed with $\mathrm{MCI}$ or dementia in the present study supports the findings of previous studies [34-36, 43, 45, 46]. Earlier findings regarding the association between depressive symptoms and independent functioning are mixed. The absence of a significant association between elevated depressive symptoms and independent functioning (IADL score) found in the present study is consistent with previous studies that reported no relationship between depressive symptoms (as assessed by the GDS-5) and IADL in patients with amnestic MCI [45] and no relationship between depression and caregiver-rated activities of daily living among patients with early-onset dementia [36]. However, our findings are in contrast to previous studies that found a positive relationship between depression and functional impairment in communitybased patients with dementia [40,43] and dementia due to AD [34].

A limitation of this study is the small sample size of the MCI patient group ( $n=51)$, which may result in an overestimation of the OR in the logistic regression analyses. Furthermore, memory clinic patients may not be representative of community-based individuals diagnosed with MCI or dementia in other settings (e.g. general or specialist practice). That is, individuals with $\mathrm{MCI}$ and depressive symptoms may be more likely to be referred to a memory clinic for diagnosis and treatment than MCI individuals without depressive symptoms. Finally, the cross-sectional design of this analysis limits interpretation of the findings to association; the direction of that association is more appropriately assessed with longitudinal data.

\section{Conclusion}

The greater prevalence and severity of elevated depressive symptoms in rural memory clinic patients with a diagnosis of MCI versus dementia (AD and non-AD) found in the present study warrant further investigation into the role of depressive symptoms in the progression of MCI to dementia [6] using longitudinal data. This investigation might focus on the possible role of depressive symptoms in increasing cognitive impairment among patients with MCI $[32,47]$. Further study is required regarding the nature and prevalence of depressive symptoms as well as different types of depression (major and minor) of varying duration and frequency (episode vs. disorder) associated with different types of MCI (amnestic, nonamnestic, or other) and dementia (AD or non-AD).

It is worth noting that elevated depressive symptoms among patients diagnosed with dementia were independently associated with lower self-rated memory and quality of life. Research consistently demonstrates a shortage of adequate primary health care and specialized services for individuals with dementia in rural and remote settings [48] and a lower usage of mental health services among rural than among urban dwellers [49]. Recent research contributes to debunking the prevailing myth that informal social networks can compensate for the lack of rural health services when most rural older adults actually lack family or friends in the community to help coordinate their care [50]. Coupled with this research, the present study provides evidence that rural older adults with MCI or dementia plus depressive symptoms constitute a vulnerable population that requires further attention and study. 


\section{References}

1 Barcelos-Ferreira R, Lopes MA, Nakano EY, Steffens DC, Bottino CMC: Clinical and sociodemographic factors in a sample of older subjects experiencing depressive symptoms. Int J Geriatr Psychiatry 2012;27:924-930.

-2 Chou KL, Chi I: Prevalence and correlates of depression in Chinese oldest-old. Int J Geriatr Psychiatry 2005; 20:41-50.

-3 Luppa M, Sikorski C, Luck T, Weyerer S, Villringer A, König HH, Riedel-Heller SG: Prevalence and risk factors of depressive symptoms in latest life: results of the Leipzig Longitudinal Study of the Aged (LEILA 75+). Int J Geriatr Psychiatry 2012;27:286-295.

4 Meeks TW, Vahia IV, Lavretsky H, Kilkarni G, Jeste DV: A tune in 'a minor' can 'b major': a review of epidemiology, illness course, and public health implications of subthreshold depression in older adults. J Affect Disord 2010;129:126-142.

5 Enache D, Winblad B, Aarsland D: Depression in dementia: epidemiology, mechanisms, and treatment. Curr Opin Psychiatry 2011;24:461-472.

-6 Panza F, Frisardi V, Capurso C, D’Introno A, Colacicco AM, Imbimbo BP, Santamato A, Vendemiale G, Seripa D, Pilotto A, Capurso A, Solfrizzi V: Late-life depression mild cognitive impairment and dementia: possible continuum? Am J Geriatr Psychiatry 2010;18:98-116.

7 Richly P, Manes F, Bustin J: Depression and Alzheimer's disease; in Kanner AM (ed): Depression in Neurologic Disorders: Diagnosis and Management, ed 1. Oxford, Blackwell, 2012, pp 177-188.

8 Petersen R: Mild cognitive impairment as a diagnostic entity. J Intern Med 2004;256:183-194.

-9 Alzheimer's Association, Thies W, Bleiler L: 2011 Alzheimer's disease facts and figures. Alzheimers Dement 2011; 7:208-244.

10 Barca M, Engedal K, Laks J, Selbaek G: Factors associated with a depressive disorder in Alzheimer's disease are different from those found for other dementia disorders. Dement Geriatr Cogn Disord Extra 2012;2:19-28.

$\checkmark 11$ Butters MA, Young JB, Lopez O, Aizenstein HJ, Mulsant BH, Reynolds CF 3rd, DeKosky ST, Becker JT: Pathways linking late-life depression to persistent cognitive impairment and dementia. Dialogues Clin Neurosci 2008; 10:345-357.

12 Pellegrino L, Peters M, Lyketsos C, Marano C: Depression in cognitive impairment. Curr Psychiatry Rep 2013; 15:384.

13 Kessing LV: Depression and the risk for dementia. Curr Opin Psychiatry 2012;25:457-461.

-14 Kasahara H, Tsumura M, Ochiai Y, Furukawa H, Aoki K, Ito T, Kada H, Hashidume T, Nakanishi T: Consideration of the relationship between depression and dementia. Psychogeriatrics 2006;6:128-133.

15 Modrego P, Ferrandez J: Depression in patients with mild cognitive impairment increases the risk of developing dementia of Alzheimer type. Arch Neurol 2004;61:1290-1293.

-16 Gao Y, Huang C, Zhao K, Ma L, Qiu X, Zhang L, Xiu Y, Chen L, Lu W, Huang C, Tang Y, Xiao Q: Depression as a risk factor for dementia and mild cognitive impairment: a meta-analysis of longitudinal studies. Int J Geriatr Psychiatry 2013;28:441-449.

17 Statistics Canada: 2011 census: population and dwelling counts Saskatoon. 2011. http://www12statcangcca/ census-recensement/2011/dp-pd/vc-rv/indexcfm?Lang=ENG\&VIEW=C\&CFORMAT=jpg\&GEOCODE=725\& TOPIC_ID=1.

18 Morgan D, Crossley M, Kirk A, D’Arcy C, Stewart N, Biem J, Forbes D, Harder S, Basran J, dal Bello-Haas V, McBain L: Improving access to dementia care: development and evaluation of a rural and remote memory clinic. Aging Ment Health 2009;13:17-30.

19 Radloff LS: The CES-D scale: a self-report depression scale for research in the general population. Appl Psychol Meas 1977;1:385-401.

20 Huba GJ, Melchior LA; Staff of the Measurement Group and HRSA/HAB's SPNS Cooperative Agreement Steering Committee: Module 26A: CES-D form (interview). 1995. http://wwwTheMeasurementGroup.com.

-21 Beekman A, Geerlings S, Deeg D, Smit J, Schoevers R, Beurs E, Braam A, Penninx B, Tilburg W: The natural history of late-life depression. Arch Gen Psychiatry 2002;59:605-611.

22 Smarr K, Keefer A: Measures of depression and depressive symptoms. Arthritis Care Res 2011;63:S454-S466.

23 Ewing JA: The CAGE questionnaire. JAMA 1984;252:1905-1907.

24 Teng E, Chui H: The Modified Mini-Mental State (3MS) Examination. J Clin Psychiatry 1987;48:314-318.

-25 Squire LR, Zouzounis JA: Self-ratings of memory dysfunction: different findings in depression and amnesia. J Clin Exp Neuropsychol 1988;10:727-738.

26 Logsdon RG, Gibbons LE, McCurry SM, Teri L: Quality of life in Alzheimer's disease: patient and caregiver reports. J Ment Health Aging 1999;5:21-32.

27 Lawton MP, Moss M, Fulcomer M, Kleban MH: A research and service oriented multilevel assessment instrument. J Gerontol 1982;37:91-99.

28 El-Masri MM, Fox-Wasylyshyn SM: Missing data: an introductory conceptual overview for the novice researcher. Can J Nurs Res 2005;37:156-171.

29 Hawthorne G, Elliott P: Imputing cross-sectional missing data: comparison of common techniques. Aust NZ J Psychiatry 2005;39:583-590.

-30 Cohen J: A power primer. Psychol Bull 1992;112:155-159.

31 Hosmer DW, Lemeshow S: Applied Logistic Regression. Toronto, Wiley, 2000. 
Kosteniuk et al.: Prevalence and Covariates of Elevated Depressive Symptoms in Rural Memory Clinic Patients with Mild Cognitive Impairment or Dementia

-32 Feldman H, Scheltens P, Scarpini E, Hermann N, Mesenbrink P, Mancione L, Teken S, Lane R, Ferris S: Behavioral symptoms in mild cognitive impairment. Neurology 2004;62:1199-1201.

-33 van der Mussele S, Bekelaar K, le Bastard N, Vermeiren Y, Saerens J, Somers N, Mariën P, Goeman J, de Deyn PP: Prevalence and associated behavioral symptoms of depression in mild cognitive impairment and dementia due to Alzheimer's disease. Int J Geriatr Psychiatry 2013;28:947-958.

34 Holtzer R, Scarmeas N, Wegesin DJ, Albert M, Brandt J, Dubois B, Hadjigeorgiou GM, Stern Y: Depressive symptoms in Alzheimer's disease: natural course and temporal relation to function and cognitive status. J Am Geriatr Soc 2005;53:2083-2089.

-35 Fritze F, Ehrt U, Sønnesyn H, Kurz M, Hortobágyi T, Nore SP, Ballard C, Aarsland D: Depression in mild dementia: associations with diagnosis, APOE genotype, and clinical features. Int J Geriatr Psychiatry 2011;26: 1054-1061.

-36 Rosness TA, Barca ML, Engedal K: Occurrence of depression and its correlates in early-onset dementia patients. Int J Geriatr Psychiatry 2010;25:704-711.

-37 Johnson L, Mauer C, Jahn D, Song M, Wyshywaniuk L, Hall J, Balldin V, O’Bryant S: Cognitive differences among depressed and non-depressed MCI participants: a project FRONTIER study. Int J Geriatr Psychiatry 2013;28: 377-382.

38 Hidaka S, Ikejima C, Kodama C, Nose M, Yamashita F, Sasaki M, Kinoshita T, Tanimukai S, Mizukami K, Takahashi H, Kakuma T, Tanaka S, Asada T: Prevalence of depression and depressive symptoms among older Japanese people: comorbidity of mild cognitive impairment and depression. Int J Geriatr Psychiatry 2011;27: 271-279.

-39 Castilla-Puentes RC, Habeych ME: Subtypes of depression among patients with Alzheimer's disease and other dementias. Alzheimers Dement 2010;6:63-69.

40 Dorenlot P, Harboun M, Bige V, Henrard JC, Ankri J: Major depression as a risk factor for early institutionalization of dementia patients living in the community. Int J Geriatr Psychiatry 2005;20:471-478.

41 Newman SC: The prevalence of depression in Alzheimer's disease and vascular dementia in a population sample. J Affect Disord 2009;52:169-176.

42 Park JH, Lee SB, Lee TJ, Lee DY, Jhoo JH, Youn JC, Choo IH, Choi EA, Jeong JW, Choe JY, Woo JI, Kim KW: Depression in vascular dementia is quantitatively and qualitatively different from depression in Alzheimer's disease. Dement Geriatr Cogn Disord 2007;23:67-73.

43 Hargrave R, Reed B, Mungas D: Depressive syndromes and functional disability in dementia. J Geriatr Psychiatry Neurol 2000;13:72-77.

44 Beattie A, Daker-White G, Gilliard J, Means R: Younger people in dementia care: a review of service needs, service provision and models of good practice. Aging Ment Health 2002;6:205-212.

45 Hudon C, Belleville S, Gauthier S: The association between depressive and cognitive symptoms in amnestic mild cognitive impairment. Int Psychogeriatr 2008;20:710-723.

46 Powlishta KK, Storandt M, Mandernach TA, Hogan E, Grant EA, Morris JC: Absence of effect of depression on cognitive performance in early-stage Alzheimer disease. Arch Neurol 2004;61:1265-1268.

47 Richard E, Reitz C, Honig L, Schupf N, Tang M, Manly J, Mayeux R, Devanand D, Luchsinger J: Late-life depression mild cognitive impairment and dementia. JAMA Neurol 2013;70:383-389.

48 Morgan D, Innes A, Kosteniuk J: Dementia care in rural and remote settings: a systematic review of formal or paid care. Maturitas 2011;68:17-33.

49 Romans S, Cohen M, Forte T: Rates of depression and anxiety in urban and rural Canada. Soc Psychiatry Psychiatr Epidemiol 2011;46:567-575.

50 Skinner MW, Rosenberg MW, Lovell SA, Dunn JR, Everitt JC, Hanlon N, Rathwell TA: Services for seniors in small-town Canada: the paradox of community. Can J Nurs Res 2008;40:80-101. 